\title{
The evolution of risk and bailout strategy in banking systems
}

\author{
Robert De Caux ${ }^{\mathrm{a}, *}$, Frank McGroarty ${ }^{\mathrm{b}}$, Markus Brede \\ ${ }^{a}$ Electronics and Computer Science, University of Southampton, Southampton, SO17 \\ 1BJ, UK. Email: rdc1g11@soton.ac.uk \\ ${ }^{b}$ Southampton Business School, University of Southampton, Southampton SO17 1BJ, \\ UK. Email: F.J.McGroarty@soton.ac.uk \\ ${ }^{c}$ Electronics and Computer Science, University of Southampton, Southampton SO17 \\ 1BJ, UK. Email: Markus.Brede@soton.ac.uk
}

\begin{abstract}
In this paper we analyse the long-term costs and benefits of bailout strategies in models of networked banking systems. Unlike much of the current literature on financial contagion that focuses on systemic risk at one point in time, we consider adaptive banks that adjust risk taking in response to internal system dynamics and regulatory intervention, allowing us to analyse the potentially crucial moral hazard aspect associated with frequent bailouts. We demonstrate that whereas bailout generally serves as an effective tool to limit the size of bankruptcy cascades in the short term, inappropriate intervention strategies can encourage risk-taking and thus be inefficient and detrimental to long term system stability. We analyse points of long-term optimal bailout and discuss their dependence on the structure of the banking network. In the second part of the paper, we demonstrate that bailout efficiency can be improved by taking into account information about the topology of and risk allocation on the banking network, and demonstrate that finely tuned intervention strategies aimed at bailing out banks in configurations with some degree of anti-correlated risk have superior performance. These results demonstrate that a suitable intervention policy may be a useful tool for driving the banking system towards a more robust structure.
\end{abstract}

Keywords: financial contagion, agent-based model, bank insolvency, evolutionary game theory, simulation, networks

$\overline{* \quad \text { Principal Corresponding author }}$

Preprint submitted to Elsevier

August 7, 2016 


\section{Introduction}

The global financial crisis of 2007-08 and the potential collapse of major banking institutions around the world left governments facing a major dilemma; should they offer financial assistance to distressed banks in the form of a bailout, or leave them to go bankrupt and face the systemic consequences for the rest of the economy. Reluctantly, many institutions were offered assistance (Rose and Wieladek, 2012; Ait-Sahalia et al., 2012), including Northern Rock, Royal Bank of Scotland and Lloyds Banking Group in the UK, with some politicians suggesting that such intervention should never be allowed to happen again (Birchler, 2014).

Although the recent example saw their use on an unprecedented scale, bailouts have been a feature of crises dating back to the 1800s (Nosal and Ordonez, 2013) and understanding their effective use is a problem that is well established in the literature. The main argument against bailing out banks is the creation of moral hazard, with empirical studies (Dam and Koetter, 2012) demonstrating that banks will take more risk if they know

they will be supported in the event of difficulty (Holmstrom and Tirole, 1998). However, a bailout can offer protection against widespread contagion, such as the panic that swept through the financial markets in the wake of Lehman Brothers' insolvency. This situation has traditionally been viewed as a tradeoff between the regulators' preference for minimising either moral hazard 
or contagion (Goodhart and Huang, 1999) and there is an extensive gametheoretic literature analysing the optimal regulatory policy (Dell'Ariccia and Ratnovski, 2012), the timing of regulatory decisions (Uhlig, 2010; Bianchi, 2012) and whether the reduction in systemic risk may outweigh the moral hazard effect (Cordella and Yeyati, 2003).

However, these models lack a detailed analysis of the true systemic risk within the interbank market, as they fail to capture network structure, heterogeneity and the fact that some banks might be Too-Big-To-Fail (Morrison, 2011; Stern and Feldman, 2004) due to the potentially catastrophic financial contagion their bankruptcy would cause (Baker and McArthur, 2009). The need for a better understanding of systemic risk (Zigrand, 2014) has led to a dramatic rise in papers analysing contagion using percolation dynamics (Nier et al., 2008; Gai and Kapadia, 2010), demonstrating that the financial system is robust-yet-fragile (Haldane, 2009), with network connectivity acting as either a means of risk diversification or a means of contagion depending on the size of the shock applied to it (Tedeschi et al., 2012; Battiston et al., 2012b). Recent models have introduced endogenously-formed dynamic interbank networks and multiple contagion channels (Bluhm et al., 2013; Georg, 2013; Aldasoro et al., 2015), which allow the market dynamics of a bankruptcy to be studied both ex ante and ex post. However, while these models can be used to calculate a systemic risk value for each bank(Battiston et al., 2012a), they are not suitable for analysing the effect of resolution policy as they assume banks' risk appetites to be fixed rather than adaptive, meaning that no moral 
hazard effect be captured in the system.

The novel aspect of the model we present is that banks within our system are adaptive, adjusting their strategy according to the success of their peers (Bala and Goyal, 1998; Gale and Kariv, 2003; Jiang et al., 2014). Accounting for this adaptability is a crucial aspect that has been missing from the contagion literature (Upper, 2011; Aymanns and Georg, 2014) and allows us to investigate the endogenous accumulation of risk (Farhi and Tirole, 2011) when banks increase their leverage (Kuzubas et al., 2014), and homogenise their asset portfolios (Allen et al., 2012) and risk management (Haldane, 2009). Banks have been shown to load up on risky assets as the system moves into danger (Daníelsson and Zigrand, 2008) and correlate their assets even though portfolio theory would suggest diversification (Beale et al., 2011).

The key to measuring the effectiveness of an intervention strategy by the regulator is a long term cost-benefit analysis (Diamond and Rajan, 2002). Our model features two dynamic processes operating at different time-scales, with slow strategy updating coupled with fast contagion dynamics in a similar manner to Battiston (2012b). This set-up allows us to model the long term dynamics of the system and the feedback between a regulator's bailout behaviour, systemic risk and economic performance. There are very few existing models that allow the long term effects of bailout policy to be investigated in a quantitative manner (Birchler, 2014; DeYoung and Reidhill, 2013; Klimek et al., 2015) and, to our knowledge, ours is the first to assess the 
effects of moral hazard and adaptive risk on bailout policy using a network contagion model.

Our approach allows us to analyse two key questions from the moral hazard and bailout literature. Firstly, we investigate the concept of "constructive ambiguity" (Freixas, 1999), where the bailout response of the regulator is purely probabilistic. Secondly, we investigate preferential or "tiered" bailout strategies (Gong and Jones, 2013), where the intervention is dependent on either the size of the distressed bank (Rose and Wieladek, 2012) or the risk level of its immediate neighbours. Both approaches are assessed across different network configurations, using a utility function that incorporates bank dividends, bankruptcy costs and bailout costs (Beale et al., 2011). This allows the overall social cost of different intervention strategies to be compared.

\section{Materials and Methods}

\subsection{Bank strategy}

We model a set of $N$ banks, each characterised by a strategy $0 \leq x_{i} \leq 1$ which determines the bank's intention to take on risk. Bank profit depends on this strategy, i.e. we set $\pi_{i}=f\left(x_{i}\right)$. We assume that risk-taking will generally result in larger (short term) profits, hence $f(\cdot)$ is assumed to be a monotonically increasing function of its argument. For simplicity we set $f(x)=x$. However, risk-taking is also associated with a higher degree of fragility, and thus we assume that a bank's likelihood of becoming bankrupt or suffering from asset write-downs is also an increasing function $g\left(x_{i}\right)$ of its 
risk-taking strategy $x_{i}$. For the purposes of this abstract model we assume that $g(x)=x$.

\subsection{Bank network}

Banks are connected via an undirected network of asset co-investments (or business relationships). A bank $i$ with $k_{i}$ network neighbours is assumed to have size $k_{i}$, i.e. the larger a bank the more asset classes it is invested in. In this sense we assume that banks' asset portfolios are maximally diversified (Battiston et al., 2012b).

Interdependency networks between banks are constructed as follows. Firstly, we consider a "regionalised" banking system, corresponding to networks given by 2D spatial grids with periodic boundary conditions and Moore neighbourhoods (one could interpret these as sets of regional assets, such that banks preferentially invest into assets in their geographical neighbourhoods). In order to consider globalisation, we build (regular) small-worlds from these networks by rewiring a fraction $\rho$ of all links. In these two scenarios, a world consisting of equally sized banks is modelled. Secondly, in order to account for heterogeneity in bank sizes and asset portfolios, we consider networks with power-law degree distributions, built either according to the preferential attachment model (Albert and Barabási, 2002) or using the configuration model to construct general random graphs with a power law degree distribution characterised by exponent $\nu$. In these models we restrict connectivity to the same number of links as for the lattice and small-world 
networks and enforce a minimum degree of one to ensure there is always one giant connected component, which comprises at least $95 \%$ of all nodes. In the case of heterogeneous networks, bank sizes are also assumed to be distributed according to a power law with exponent $\nu$.

\subsection{Systemic shocks}

Two causes for insolvency are considered: (i) External fluctuations: We assume that with a small likelihood $p_{0}$ an event will occur that puts a bank into distress, and that the bank will become insolvent with probability $x_{i}$ directly influenced by its risk-taking strategy. An insolvent bank is bankrupt at this point if it is not bailed out; (ii) Contagion of adverse effects: Once a bank becomes bankrupt due to an external fluctuation in (i), there is a small probability $\mu=0.01$ that the bankruptcy process is disorderly and reduces the price of its assets, causing contagion via the network of asset co-investments (Furfine, 2003). If this is the case, banks that are network neighbours of the insolvent bank will suffer a shock. With probability $\max \left(\eta / k_{i}, 1\right)$, this shock will now cause insolvency in bank $i$ (i.e. we assume that larger banks with more diversified asset portfolios are more resilient to shocks than smaller banks), putting this new bank's respective network neighbours in distress and potentially causing a cascade of further insolvencies. After bankruptcy, a bank reverts to strategy $x=0$ and resumes its former place in the banking network. In the case of a shock that does not cause insolvency, which occurs with probability $1-\max \left(\eta / k_{i}, 1\right)$, the shock results in an asset write-down 
and a bank will adjust its risk-taking strategy to adapt to the loss, i.e. we set $x_{i} \rightarrow x_{i}(1-\eta / k)$ (again, assuming that smaller banks react with more drastic adjustments than larger banks which are more diversified). The parameter $\eta$ models the relative likelihood that shocks will result in asset write-down or insolvency, with $\eta=\max _{i} k_{i}$ corresponding to a scenario in which distress always causes insolvency, and $\eta=0$ modelling a scenario in which all distress may at most cause asset write-downs (and corresponding strategy adjustments). Assuming $<k>=8$ for our simulations, we used $\eta=4$, with larger values giving similar results and smaller values not allowing cascades to percolate.

\subsection{Bailout}

We also model the effects of (government) bailouts. Once a bank is distressed, it will be bailed out with probability $q$, thus preventing a bankruptcy. The bank resumes normal trading using its previous risk-taking strategy and distress does not spread to its business partners. In the UK, the size of the bank relative to the entire banking system has a strong correlation with the probability of a bailout (Rose and Wieladek, 2012). As a refinement of this described mixed strategy bailout, two further bailout strategies are considered: (i) size-based bailout, and (ii) neighbour-dependent bailout. In the first case, banks with size (or degree) larger than a threshold $k$ will be bailed out when distressed, whereas smaller banks will not be. In the case

of neighbour-dependent bailout, a bank $i$ will be bailed out with likelihood 
$q_{i}=c_{0}+c_{1} k_{i}^{-\theta} \sum_{j} a_{i j} x_{j}$ where $c_{0}, c_{1}$, and $\theta$ are parameters characterising this bailout strategy and $a_{i j}$ is the adjacency matrix of the network. When investigating strategy (ii), we search the parameter space spanned by $c_{0}, c_{1}$ and $\theta$ to find optimal interventions.

\subsection{Evolution of risk}

To investigate the dynamics of the evolution of risk-taking, we consider two processes:

- Risk-taking strategy adaptation: At each time step a randomly chosen bank, say bank $i$, adapts its risk-taking strategy by comparing its profits to a randomly selected other bank of similar size, e.g. bank $j$, allowing for choices of reference banks with $\pm 30 \%$ size relative to the bank $i$ that is updating its strategy (Bala and Goyal, 1998; Gale and Kariv, 2003; Jiang et al., 2014). If $j$ achieved a higher profit, bank $i$ will adapt to bank $j$ 's strategy $x_{j}$ by adjusting its risk-taking strategy in proportion to the difference to $j$, i.e. $x_{i} \rightarrow x_{i}+\sigma\left(x_{j}-x_{i}\right)$. To allow for the evolution of novel strategies, bank $i$ 's strategy will also mutate by a small amount each turn. This is modelled by adding a small random number drawn from the uniform distribution over $\left[-p_{\mathrm{mut}}, p_{\mathrm{mut}}\right]$ (Note that strategies are always restricted to the interval $[0,1]$.) In our simulations we use $p_{\text {mut }}=0.0005$ and $\sigma=0.01$.

- Contagion of distress: With a (small) probability $p_{0}$, a randomly chosen bank, say bank $i$, is said to be in distress. With probability $x_{i}$ bank 
$i$ goes bankrupt, and, as described above, with a small probability $\mu$ distress may spread to the bank's network neighbours who might, in turn, go bankrupt, and so on. We assume that this process occurs at a timescale much faster than the evolution of strategies, resolving bankruptcies instantaneously. After a bankruptcy a bank returns to trading with strategy $x=0$.

The dynamics of the evolution of risk are thus marked by two processes (i) adaptation of bank's strategies towards risk-taking in such a way that riskier strategies are favoured (and would eventually dominate the population without the second process), and (ii) the occasional occurrence of (initially externally caused) distress that may translate into cascades of bankruptcies or asset write-downs that can potentially spread through the banking system. Lower risk-taking strategies are more likely to escape cascades of bankruptcies (and asset write-downs), hence a balance between low and high risk-taking evolves in the population.

\subsection{Measuring social cost}

In each time period, costs and benefits are determined as follows. If bank $i$ goes bankrupt, the cumulative cost of bankruptcy $C_{b}$ is incremented by a cost proportional to bank $i$ 's balance sheet, which we define as $x_{i} k_{i}$. The balance sheet is proportional to both the network degree and risk level of bank $i$. Similarly, a bailout will increment the cumulative cost of intervention $C_{v}$ by a cost in proportion to the distressed bank's balance sheet. If bank $i$ suffers 
an asset write-down through contagion, the cumulative asset write-down $C_{a}$ is incremented by $x_{i}$, representing the fact that $i$ loses $1 / k_{i}$ of its balance sheet. On the benefits side, higher risk-taking leads to larger returns for shareholders. Therefore the cumulative benefit $B$ is incremented by $\sum_{i} x_{i} k_{i}$

each time period, where $i$ runs over the set of all banks. Averaging costs and benefits over simulated time (after a transient has elapsed), a total social cost function can be determined via

$$
U(\Psi)=-C_{b}(\Psi)-C_{a}(\Psi)-\alpha C_{v}(\Psi)+\beta B(\Psi)
$$

with parameters $\alpha$ and $\beta$ determining the relative costs of bankruptcy, bailout and shareholder dividends from banks. For the purposes of this abstract model, we set $\alpha=10$ under the assumption that bailout is far more costly than bankruptcy, and we set $\beta=0.01$ assuming a relatively small risk related return per unit of time for banks. Note that the social cost function depends on $\Psi$, which is the choice of intervention strategy.

\section{Results and Discussion}

\subsection{Self-organised criticality}

Previous work has likened financial networks to a self-organised critical (SOC) system (Hurd, 2015) and we have built on those ideas in the development of our stylised model. In the absence of shocks, banks adapt their strategies to those of economically more successful competitors, leading to 
an accumulation of risk on a slow time-scale and making individual banks vulnerable to failure. The potential for cascades of bankruptcies reduces the rewards of risky strategies; hence risk-taking balances out and is marked by cycles of larger and smaller risk appetite of banks that correspond to fluctuations around some intermediate level of risk.

Fig. 1 illustrates these SOC-like dynamics. It shows the existence of periods of calm, marked by the accumulation of risk, which are interrupted by cascading shocks of various sizes. Cascades of failures are mostly small and localised to only very few banks, but can occasionally reach system-wide proportions, cf. panel (C) of Fig. 1 which gives the distribution of the sizes of simulated bankruptcy cascades. Similar to models of the long-term dynamics of forest fires, the model exhibits power law distributed cascades and SOClike behaviour in the limit of extremely rare external shocks (Drossel and Schwabl, 1992; Pruessner and Jensen, 2002). In the case of more frequent shocks the distribution is bimodal, with peaks for very small cascades and rare very large cascades.

\subsection{Utilising constructive ambiguity}

More importantly, the model allows us to investigate the system's longterm response to interventions by a regulator. Fig. 2 shows the dependence of average risk-taking on a regulator's "constructively ambiguous" bailout strategy, where $q$ represents the probability of intervention. In agreement with empirical evidence (Dam and Koetter, 2012), we find that larger prob- 
$(A)$

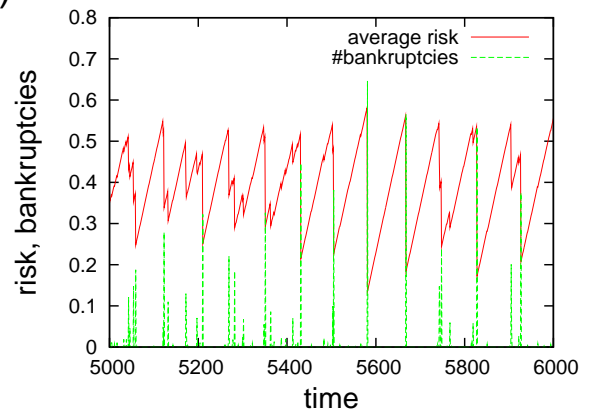

(B)

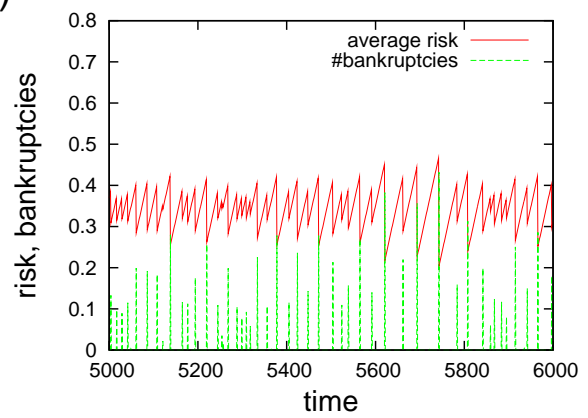

(C)

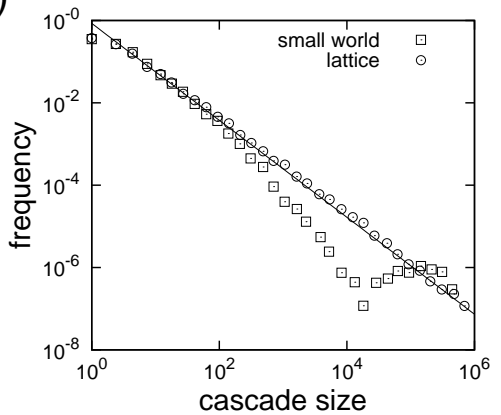

Figure 1. Evolution of risk and bankruptcies. Results shown on (A) a lattice and (B) a small-world network, with (C) corresponding distributions of bankruptcy cascades. Systems are of size $1.44 \times 10^{6}$ nodes with an average degree $\langle k\rangle=8$, a probability of exogenous shocks $p_{0}=10^{-4}$ and a rewiring probability for the small-world network $\rho=0.05$.

abilities of bailout will enhance a bank's average appetite for risk. However, this enhanced risk-taking potentially makes the system more fragile, which may cause relatively more failures than in a scenario without regulatory intervention. As a consequence, the long-term repercussions of bailout may actually be detrimental to overall system function. Fig. 3 illustrates this behaviour by comparing the average number of banks affected by cascades (i.e. the number of bankrupt banks and bailed-out banks) to the bailout strategy in both the short and long term for prototypical classes of asset correlation network. In the short term, before banks have had the chance to adapt their 
strategies, the response to bailout is positive; large numbers of bailed-out banks reduce the size of bankruptcy cascades, resulting in a monotonic decline of $n_{\text {bailout }}+n_{\text {bankrupt }}$ with the bailout probability $q$. At some critical point a percolation threshold $q_{\text {perc }}$ is reached, such that for $q>q_{\text {perc }}$, systemwide cascades can be prevented. This contrasts with the long-term response to enhancing bailout likelihood, which is not monotonic. Crucially, increasing the intervention probability from zero results in a more vulnerable system prone to typically larger cascades of failures. This trend is only reversed at some point shortly before the percolation threshold is reached. Similarly to the short-term scenario, increasing $q$ above $q_{\text {perc }}$ suppresses system-wide failure, but enhances risk-taking and leads to a large number of bailouts that may be socially inefficient. Hence, typically a bailout probability $0<q<1$ exists such that the overall additional profit available through increased bank risk is optimally balanced against the cost of failures and bailouts.

(A)

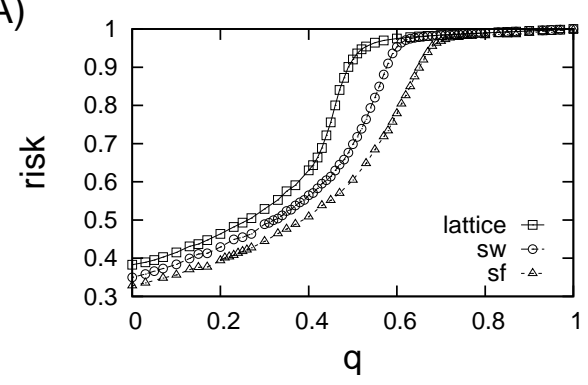

(B)

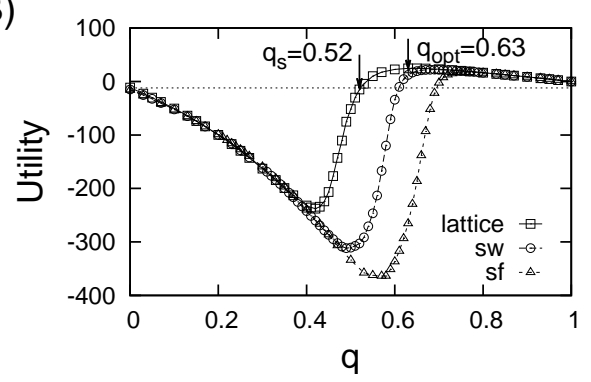

Figure 2. Bank risk and utility. Average (A) risk and (B) system utility achieved per turn for $10^{4}$ banks, coupled by either a lattice, small world or scale free network with average degree $\langle k\rangle=8$. Results have been averaged over at least 10 network realisations, for each of which the dynamics have been run for $10^{5}$ sweeps. The arrows indicate the optimal $\left(q_{\text {opt }}\right)$ and minimum economically sensible $\left(q_{s}\right)$ bailout probabilities for a lattice.

To address this question of optimal bailout, we investigated extremal 

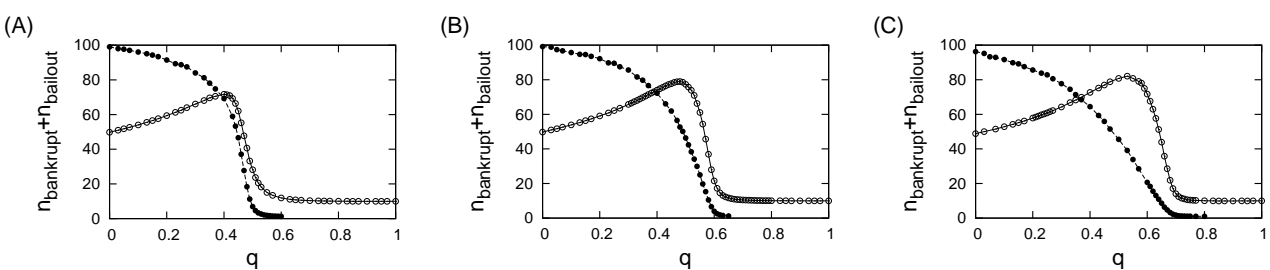

Figure 3. Influence of bailout. Comparison in the short (non-adaptive banks, solid circles) and long term (adaptive banks, open circles) quantified by plots of the value of assets bailed out and lost in bankruptcies vs. the bailout probability $q$. Simulations run for (A) lattices, (B) small-world and (C) scale-free networks.

points of a social cost-benefit function. Bank dividends are offset against bankruptcy and bailout costs, with dividend size increasing monotonically with bank risk (see Materials and Methods). Typical cost-benefit functions for various types of asset correlation network are illustrated in Fig. 2(B). In agreement with the above arguments we find that these functions are initially declining, reflecting bank's enhanced risk-taking in response to sub-critical probabilities of bailout. As the cascade limiting effect of larger degrees of intervention can exceed enhanced long-term risk-taking, this trend is reversed at some point and one typically finds a minimum economically sensible bailout probability $q_{s}$ at which the utility value achieved is the same as that for a system with no intervention. Increasing the likelihood for bailout further, there exists some probability $q_{\mathrm{opt}}>q_{\text {perc }}$ for which utility is optimised, but even though a localised spread of failures is increasingly suppressed, the costs of bailout typically become excessive beyond this point and utility begins to decline. For the three prototypical types of asset correlation networks, we find $q_{\mathrm{opt}}=0.63 \pm 0.01, q_{\mathrm{opt}}=0.67 \pm 0.01$, and $q_{\mathrm{opt}}=0.75 \pm 0.01$ for lattices, 2 dimensional small-worlds, and scale-free networks of average degree $\langle k\rangle=8$ 
respectively. The exact location of these points depends on the choice of weights in the cost-benefit function, but the maxima are typically very flat and results are robust over a wide range of cost parameters.

\subsection{Dependence on network structure}

We have also investigated the dependence of optimal bailout strategy on the structure of the asset correlation network. In a first experiment, we compare worlds of regionally localised asset investments to increasingly globalised worlds building asset correlation networks using a variant of Watts and Strogatz's small-world model (1998). As expected, one finds that the dynamics of insolvency cascades on increasingly globalised worlds are more difficult to control (see panels of Fig. 4). With no bailout, average utility declines with the shortcut density (a proxy measure of globalisation in the small-world model). In a similar vein, the minimum economically sensible bailout probability $q_{\mathrm{s}}$ and optimal bailout probability $q_{\mathrm{opt}}$ both increase, but utilities at the point of optimal intervention decrease. We also calculate the bailout probability $q_{\min }$ for which system utility is minimised to demonstrate how poorly the system can perform without carefully controlled intervention.

In a second experiment, we investigate the effects of system heterogeneity by constructing asset correlation networks with power-law degree distributions. The exponent of the power law, $\nu$, can be tuned to achieve the level of heterogeneity required in the network. Results are summarised in Figure 5 in which the dependence of utility, minimum economically sensible 
(A)

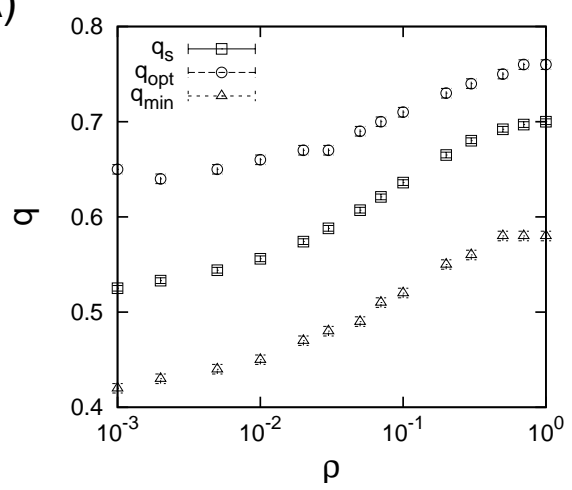

(B)

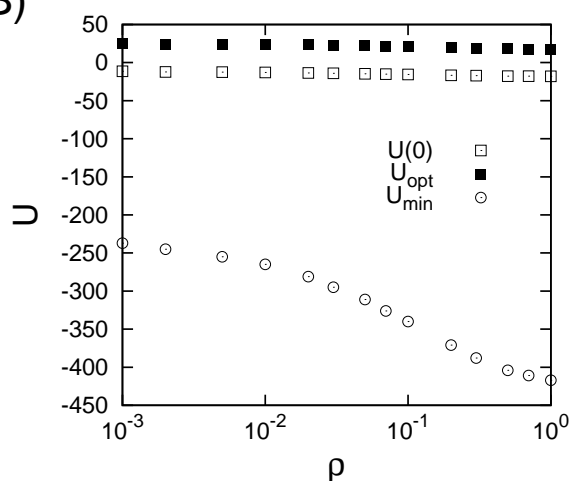

Figure 4. Regionalisation vs. globalisation. Dependence of the cost curve on the shortcut density $\rho$ of the small-world model: (A) thresholds for the minimum probability at which the bailout becomes profitable $\left(q_{\mathrm{s}}\right)$, optimal bailout probability in terms of utility $\left(q_{\mathrm{opt}}\right)$ and probability at which the utility from bailout is minimised $\left(q_{\min }\right)$; (B) utilities without intervention $(U(0))$, with the worst possible intervention probability $\left(U_{\min }\right)$ and with the optimal bailout probability $\left(U_{\mathrm{opt}}\right)$.

bailout probability and optimal bailout probability on $\nu$ are shown. We find that utilities, both with and without optimal intervention, decline as heterogeneity is decreased. Whereas the dependence of non-intervention utility on system structure is fairly strong, utilities at the maximum of the cost benefit function are broadly independent of $\nu$. As a result, there is a threshold level of system heterogeneity at which intervention becomes sensible. Within our stylised framework, we find that for systems more heterogeneous than $\nu \approx 1.9$, a mixed bailout strategy is not economically viable. For $\nu>1.9$, as systems become less heterogeneous, the bailout probability at the point of optimal intervention increases slightly with $\nu$.

\subsection{Preferential Intervention}

So far, we have considered mixed bailout strategies that do not make use of information regarding the size or position of banks in the network. What 
(A)

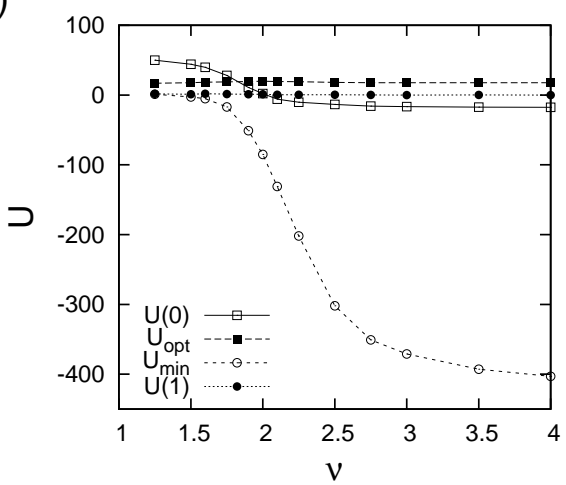

(B)

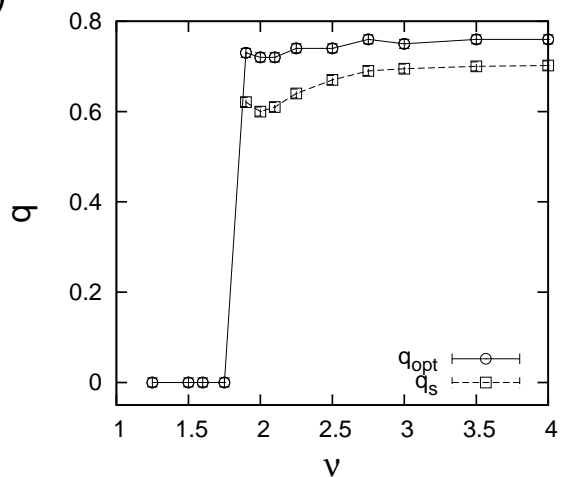

Figure 5. Effect of system heterogeneity. (A) Dependence of utility on the heterogeneity of the banking system controlled by the exponent $\nu$, shown without intervention $(U(0))$, with maximal intervention $(U(1))$, with optimal intervention $\left(U_{\text {opt }}\right)$ and worst intervention $\left(U_{\min }\right)$. (B) Dependence of the minimum economically sensible bailout probability $\left(q_{s}\right)$ and optimal bailout probability $\left(q_{\text {opt }}\right)$ on $\nu$.

about a "tiered" or preferential intervention strategy for banks that are either too connected or Too-Big-To-Fail? (Morrison, 2011; Battiston et al., 2012a) In the present model, both a bank's size and connectivity are approximated by a node's degree; hence we consider bailout strategies in which nodes of degree greater than a certain threshold $k$ are always bailed out, while other nodes are allowed to fail. In Fig. 6, the dependence of utility on $k$ is given for systems of varying heterogeneity parametrised by the exponent of the degree distribution $\nu$. The extremes of very stringent (very large $k$ ) or very lenient (very small $k$ ) bailout correspond to the extremes of $q=0$ and $q=1$ in the mixed strategy bailout scenario, which are not optimal unless system heterogeneity exceeds the threshold of $\nu \approx 1.9$ when no bailout is cost efficient (as in the case of $\nu=1.6$ displayed in the figure). In between these extremes, the dependency is generally S-shaped with extremal points at low degree and high degree, illustrating the potential benefits and (long term) dangers of 
preferentially bailing out large banks. The first maximum corresponds to a low degree threshold, which allows sufficient hub nodes to be bailed out that bankruptcy cascades can effectively be suppressed. Comparison with mixed strategy bailout shows that targeted intervention is potentially beneficial, if the bailout threshold is properly calibrated. In contrast, and similarly to the results observed for mixed strategy intervention above, there exists a threshold region where bailout encourages excessive risk-taking without being able to suppress systemic effects, and system utility is negative. This again highlights the danger that a poorly calibrated intervention strategy can have on system performance.

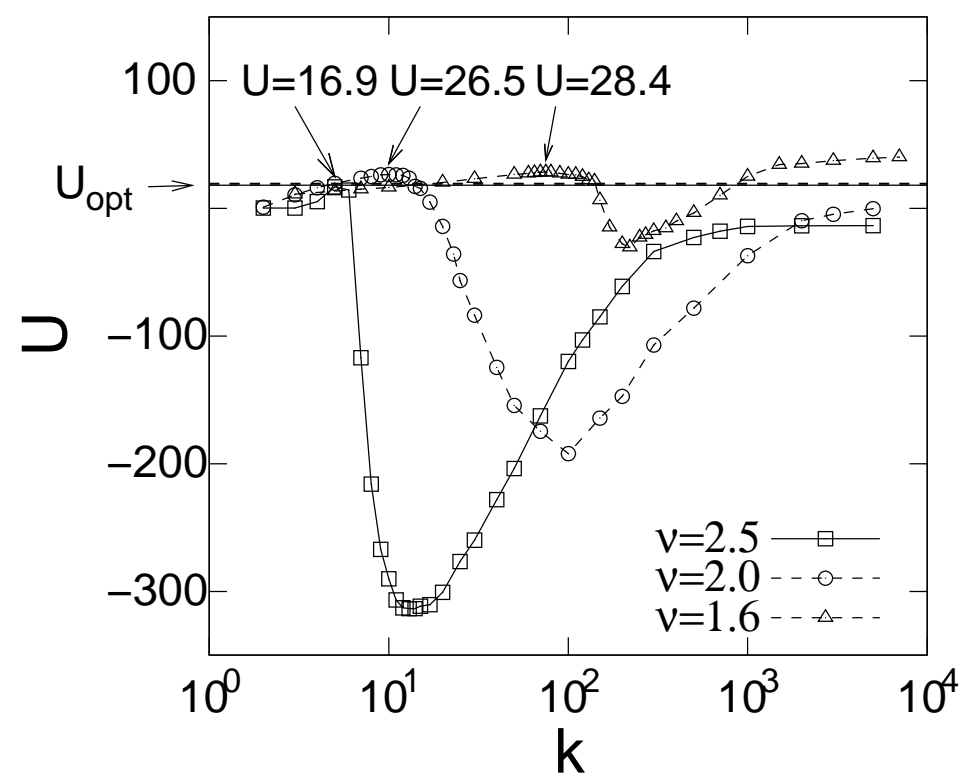

Figure 6. Effect of bank connectivity. Dependence of utility on the minimum degree $k$ for defining hub nodes for preferential bailout. Results for scale-free networks with degree exponent $\nu=2.5,2$ and 1.6. The horizontal line $U_{\text {opt }}$ gives the optimal achievable utility for a mixed strategy bailout that does not use topological information.

As a refinement of degree-based bailout, we also consider a class of in- 
tervention strategies that select whether to bailout banks dependent on the riskiness of their neighbours' and their consequent susceptibility to being impacted by a bankruptcy cascade. Rather than $q$ being a "static" probability as it was previously, it is now determined by a linear function of total neighbour risk, where $x_{j}$ represents a bank strategy and $a_{i j}$ the adjacency matrix for the network:

$$
q_{i}=c_{0}+c_{1} f_{i} \sum_{j} a_{i j} x_{j}
$$

with coefficients $c_{0}$ and $c_{1}$ as parameters and node-dependent coefficients $f_{i}=k_{i}^{-\theta}$ potentially allowing for size-specific factors such that this class of strategies can be seen as a superset of the size-based interventions presented above (see Materials and Methods). In a static setting without adaptive risktaking, we would expect preferential bailout of positively correlated risk, i.e. positive coefficients $c_{1}$, to be the most efficient way of stopping bankruptcy cascades. We have searched the space of bailout strategies parametrised by $c_{0}, c_{1}$ and $\theta$ in the setting with adaptive risk and generally find utility maxima for $\theta \approx 1$, whose dependence on $c_{1}$ is shown in Fig. 7 for the three prototypical asset correlation network types.

Contrary to our intuition for the static case, we find that optimal utilities decrease sharply for positive $c_{1}$ and note a marked improvement until saturation for negative $c_{1}$. Closer inspection of the optimal strategies reveals that utility maxima are reached when banks are only bailed out if at least 
(A)

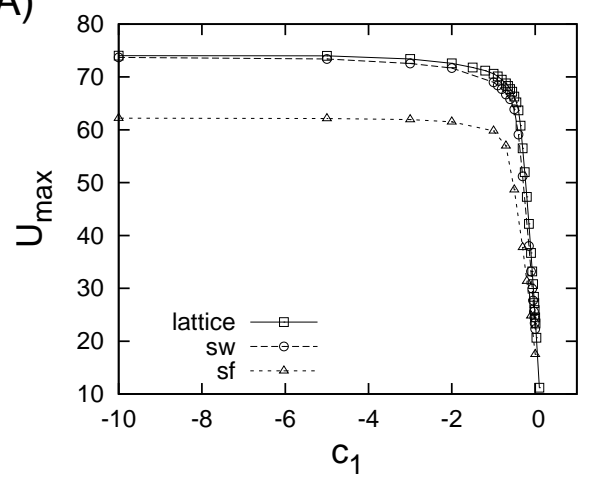

(B)

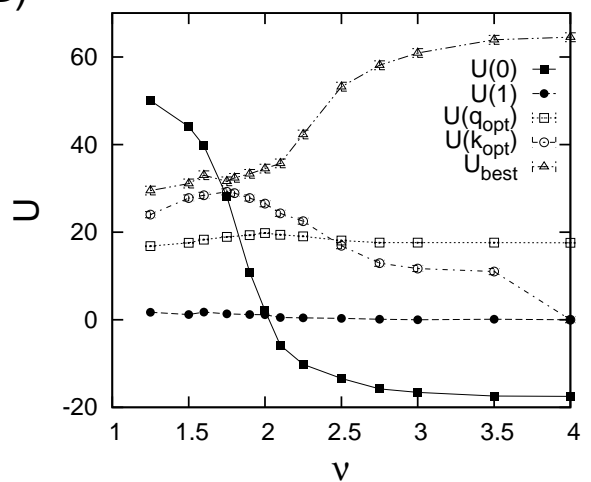

Figure 7. Neighbourhood risk. (A) Dependence of maximum achievable utility on the risk correlation factor $c_{1}$, which we find in the class $f_{i}=k_{i}^{-\theta}$ for $\theta \approx 1$. (B) Dependence of utility on system heterogeneity, parametrised by $\nu$. We compare utilities with no intervention $(U(0))$ and full intervention $(U(1))$, as well as the best mixed strategy $\left(q_{o p t}\right)$, degree-based $\left(k_{o p t}\right)$ and neighbour-based $\left(U_{\text {best }}\right)$ intervention methods.

one or two of their network neighbours take on low risks. To understand why these strategies are optimal in an adaptive setting, one should consider bailout strategies as a selection mechanism that shapes the allocation of risk. If we preferentially bail out patches of positively correlated risk, this positively correlated risk is selected for, and will eventually result in a longterm system configuration with an agglomeration of such patches requiring a large number of bailouts each turn. Conversely, negatively correlated bailout might not be beneficial in the short term, but will encourage slightly negatively correlated risk allocations, which are just enough to stop system-wide cascades with minimal bailout requirement. This can be illustrated well in spatial networks, cf. Fig. 8, which give snapshots of example configurations evolved using various bailout regimes and show the corresponding landscapes of likelihoods to stop bailout. It can be seen that in the optimal regime, a relatively large fraction of evenly distributed low risk-takers is encouraged 
who effectively restrict potential bankruptcy cascades to very small sizes.

(A)
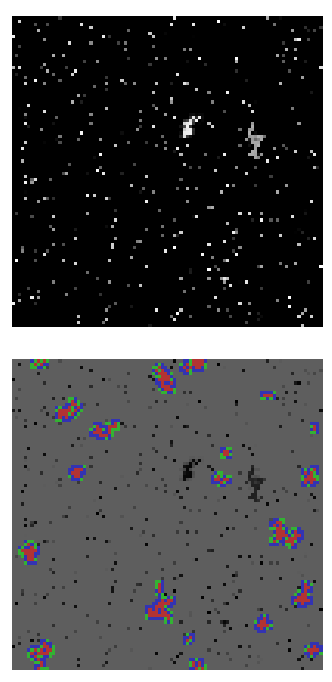

(B)

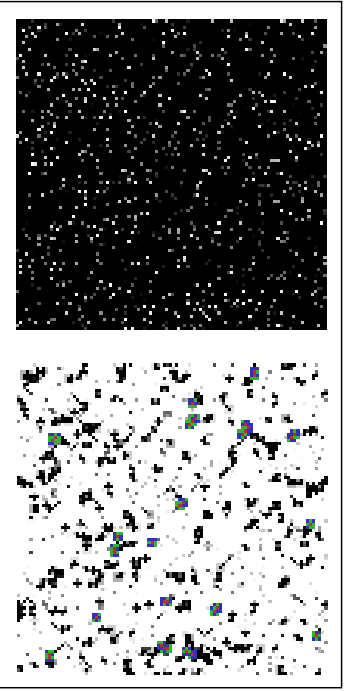

(C)

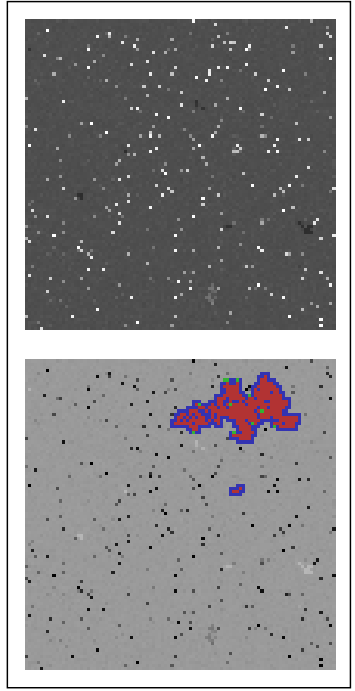

Figure 8. Risk allocation and cascades. Example configurations of risk allocation (top) and illustration of bailout likelihoods and of example cascades (bottom) for various bailout strategies. In the top pictures, darker colours indicate higher risk. In the bottom pictures, darker colours indicate a high likelihood that a bankruptcy cascade will be stopped at this bank. Example cascades started at randomly selected locations are also shown here, where red illustrates bankrupt banks, green bailed-out banks and blue banks who were affected, but survived. (A) Configuration for the optimal mixed bailout strategy $q=0.63$, (B) configuration for optimal neighbour-dependent strategy and (C) mixed bailout strategy with the same effective bailout likelihood $q=0.13$ as for the optimal neighbour dependent strategy in (B).

In Fig. 7 we also compare the dependence of utility using an optimal neighbour-dependent bailout strategy on system heterogeneity. Similarly to lattices and small-worlds, utility is greatest for large $\nu$, but decreases strongly when system heterogeneity is increased. Fig. 7 also includes a comparison of all considered types of bailout strategy for systems with varying degree of heterogeneity. We note that an (anti-correlated) neighbour-dependent bailout strategy is always superior to degree-based or mixed strategy intervention policy, but the threshold of system heterogeneity when any form of intervention becomes efficient is nearly independent of the choice of strategy. 
Closer analysis gives $\nu \approx 1.9$ for an optimal mixed strategy bailout, and a slightly lower $\nu \approx 1.8$ for degree-based and neighbour-dependent bailout (cf. intersection of the $U(0)$-curve with the curves denoting the various bailout strategies). In addition, a second crossing point at $\nu \approx 2.5$ is notable when mixed strategy bailout becomes superior to degree-based bailout. At this point, degree thresholds have to be set such that almost all nodes are bailed out all the time and choices of mixed strategy bailout allow for a finer tuning of bailout strategy.

\section{Conclusions}

In this paper we have introduced a model of adaptive risk based on a stylised banking systems, in which distress and insolvency can propagate via networks of asset co-investment. We have argued that the bailout strategies of an external regulator can be understood as a selective force that shapes the allocation of risk in this networked system, and will thus influence performance measures and system fragility. Analysing adaptive risk-taking highlights the potentially problematic effects of bailout over a sustained period of time. We provide quantitative evidence that whereas bailout will always restrain the systemic effects of insolvency in the short term, it can encourage excessive risk-taking and thus be detrimental to long term system efficiency. A long term cost-benefit analysis of bailout allows the determination of optimal intervention probabilities, and we generally find these points located in a regime above the percolation threshold of the banking network. 
In agreement with intuition, more globalised worlds require more bailout, and are harder to control than regionalised banking systems without long range connections. Furthermore, depending on the likelihood of distress situations, an optimally tuned intervention strategy is found to be always efficient in systems with minimal heterogeneity in bank size, but non-intervention can become a superior strategy if system heterogeneity exceeds some threshold.

An intervention strategy may be refined to become conditional on a bank's size or position within the network. Size-based bailouts can become superior to purely probabilistic bailouts in heterogeneous systems, if intervention thresholds are chosen such that systemic effects are suppressed but unnecessarily frequent bailout is avoided. However, we find that intervention strategies conditional on the risk-taking of a bank's neighbours are comfortably superior to probabilistic or size-based bailout. If finely tuned, such a bailout policy can favourably partition the system into regions of high risk-taking separated by boundaries of low risk-taking, thus restraining the possible size of bankruptcy cascades. The long-term optimality of bailing out (slightly) negatively correlated risk-takers may appear counter-intuitive. However, understanding that bailout can be a selective force that shapes system structure, it becomes clear that a targeted bailout strategy can encourage patterns of the same risk-taking it intends to mitigate when preventing cascades. Thus, while it is not the most efficient method of cascade prevention, bailing out banks with negatively correlated risk-taking can promote an overall system structure that enhances robustness. 
Our model intentionally uses a highly abstracted banking network to illustrate the differences between various bail-out strategies in the short and long term. Therefore, a potential area of future research would be to improve realism by extending the work to include a more detailed representation of contagion channels, e.g. based on the recent literature on multiplex networks (Montagna and Kok, 2013; Brummitt and Kobayashi, 2015; Poledna et al., 2015). Another possible avenue for future work would be to allow bank connections to evolve over time as strategies change, creating an adaptive network of interconnected balance sheets.

\section{Acknowledgements}

This work was supported by an EPSRC Doctoral Training Centre grant [EP/G03690X/1].

\section{References}

Ait-Sahalia, Yacine, et al. (2012). "How to Stop a Herd of Running Bears? Market Response to Policy Initiatives during the Global Financial Crisis". Journal of International Economics, 87(1), 162-177.

Albert, R and A.-L. Barabási (2002). "Statistical mechanics of complex networks". Rev. Mod. Phys., 74(January), 47.

Aldasoro, Inaki, Domenico Delli Gatti, and Ester Faia (2015). "Bank Net- 
works: Contagion, Systemic Risk and Prudential Policy". SAFE Working Paper, 87, 1-43.

Allen, Franklin, Ana Babus, and Elena Carletti (2012). "Asset Commonality, Debt Maturity and Systemic Risk". Journal of Financial Economics, 104, $519-534$.

Aymanns, Christoph and Co Pierre Georg (2014). "Contagious synchronization and endogenous network formation in financial networks". Journal of Banking and Finance, 50, 273-285.

Baker, D and T McArthur (2009). "The value of the "too big to fail" big bank subsidy". CEPR Reports and Issue Briefs, 36, 1-5.

Bala, Venkatesh and Sanjeev Goyal (1998). "Learning from neighbors". The Review of Economic Studies, 65(3), 595-621.

Battiston, Stefano, et al. (2012a). "DebtRank: Too Central to Fail? Financial Networks, the FED and Systemic Risk". Scientific Reports, 2, 1-6.

Battiston, Stefano, et al. (2012b). "Liaisons dangereuses: Increasing connectivity, risk sharing, and systemic risk". Journal of Economic Dynamics and Control, 36(8), 1121-1141.

Beale, Nicholas, et al. (2011). "Individual versus systemic risk and the Regulators Dilemma". PNAS, 108(31), 12647-12652.

Bianchi, Javier (2012). "Efficient Bailouts?" NBER Working Paper, 18587. 
Birchler, Urs Walter (2014). "'Never again!" - the dynamics of bank bailouts". University of Zurich Working Paper, pages 1-26.

Bluhm, Marcel, Ester Faia, and Jan Pieter Krahnen (2013). "Endogenous banks' networks, cascades and systemic risk". SAFE Working Paper Series, 12 .

Brummitt, Charles D. and Teruyoshi Kobayashi (2015). "Cascades in multiplex financial networks with debts of different seniority". Physical Review E, 91(6), 062813.

Cordella, Tito and Eduardo Levy Yeyati (2003). "Bank bailouts: Moral hazard vs. value effect". Journal of Financial Intermediation, 12(4), 300330.

Dam, Lammertjan and Michael Koetter (2012). "Bank Bailouts and Moral Hazard: Evidence from Germany". The Review of Financial Studies, 25(8), $2343-2380$.

Daníelsson, Jón and Jean Pierre Zigrand (2008). "Equilibrium asset pricing with systemic risk". Economic Theory, 35(2), 293-319.

Dell'Ariccia, Giovanni and Lev Ratnovski (2012). "Bailouts, Contagion, and Risk-Taking". Society for Economic Dynamics Meeting Paper, 133, 1-18.

DeYoung, Robert and Jack Reidhill (2013). "A Theory of Bank Resolution: Political Economics and Technological Change". Journal of Financial Stability, 9, 612-627. 
Diamond, Douglas W. and Raghuram G. Rajan (2002). "Bank Bailouts and Aggregate Liquidity". American Economic Review, 92(2), 38-41.

Drossel, B. and F. Schwabl (1992). "Self-organized critical forest-fire model". Physical Review Letters, 69(11), 1629-1632.

Farhi, Emmanuel and Jean Tirole (2011). "Collective moral hazard, maturity mismatch and systemic bailouts". American Economic Review, 102(1), 6093.

Freixas, Xavier (1999). "Optimal Bail Out Policy, Conditionality and Constructive Ambiguity". CEPR Discussion Paper, 2238.

Furfine, Craig H (2003). "Interbank Exposures: Quantifying the Risk of Contagion". Journal of Money, Credit and Banking, 35(1), 111-128.

Gai, Prasanna and Sujit Kapadia (2010). "Contagion in financial networks". Proceedings of the Royal Society of London A: Mathematical, Physical and Engineering Sciences, 383.

Gale, Douglas and Shachar Kariv (2003). "Bayesian learning in social networks". Games and Economic Behavior, 45(2), 329-346.

Georg, Co Pierre (2013). "The effect of the interbank network structure on contagion and common shocks". Journal of Banking and Finance, 37(7), $2216-2228$. 
Gong, Ning and Kenneth D. Jones (2013). "Bailouts, monitoring, and penalties: An integrated framework of government policies to manage the toobig-to-fail problem". International Review of Finance, 13(3), 299-325.

Goodhart, Charles A E and Haizhou Huang (1999). "A Model of the Lender of Last Resort". IMF Working Paper, 39, 1-33.

Haldane, Andrew G (2009). "Rethinking the financial network". Bank of England speech, delivered at Financial Student Association, Amsterdam, Netherlands, April, 1-41. URL http://www. bankofengland.co.uk/ archive/Documents/historicpubs/speeches/2009/speech386.pdf.

Holmstrom, Bengt and Jean Tirole (1998). "Private and Public Supply of Liquidity". Journal of Political Economy, 106(1), 1-40.

Hurd, T R (2015). Contagion! The Spread of Systemic Risk in Financial Networks. Springer, forthcomin edition.

Jiang, X F, T T Chen, and B Zheng (2014). "Structure of local interactions in complex financial dynamics." Scientific reports, 4, 1-9.

Klimek, Peter, et al. (2015). "To bail-out or to bail-in? Answers from an agent-based model". Journal of Economic Dynamics and Control, 50, 144154 .

Kuzubas, Tolga Umut, Burak Saltoglu, and Can Sever (2014). "Systemic Risk and Heterogeneous Leverage in Banking Network: Implications for Bank- 
ing Regulation". Bogazici University Department of Economics Working Paper, 1, 1-28.

Montagna, Mattia and Christoffer Kok (2013). "Multi-layered Interbank Model for Assessing Systemic Risk". Kiel Working Paper, 1873.

Morrison, Alan D. (2011). "Systemic risks and the 'too-big-to-fail' problem"”. Oxford Review of Economic Policy, 27(3), 498-516.

Nier, Erlend, et al. (2008). "Network models and financial stability". Bank of England Working Paper, 346.

Nosal, Jaromir and Guillermo Ordonez (2013). "Uncertainty as commitment". NBER Working Paper, 18766, 1-46.

Poledna, Sebastian, et al. (2015). "The multi-layer network nature of systemic risk and its implications for the costs of financial crises". Journal of Financial Stability, 20, 70-81.

Pruessner, Gunnar and Henrik Jeldtoft Jensen (2002). "Broken scaling in the Forest Fire Model". Physical Review E, 65(5), 9.

Rose, Andrew K and Tomasz Wieladek (2012). "Too big to fail: some empirical evidence on the causes and consequences of public banking interventions in the United Kingdom”. Bank of England Working Paper, 460.

Stern, Gary H and Ron J Feldman (2004). Too Big To Fail: The Hazards of Bank Bailouts. Brookings Institution Press, Washington DC. 
Tedeschi, Gabriele, et al. (2012). "Bankruptcy cascades in interbank markets." PloS one, 7(12), 1-10.

Uhlig, Harald (2010). "A model of a systemic bank run". Journal of Monetary Economics, 57(1), 78-96.

Upper, Christian (2011). "Simulation methods to assess the danger of contagion in interbank markets". Journal of Financial Stability, 7(3), 111-125.

Watts, D.J. J and S.H. H Strogatz (1998). "Collective dynamics of smallworld networks". Nature, 393(6684), 440-442.

Zigrand, Jean-Pierre (2014). "Systems and Systemic Risk in Finance and Economics". London School of Economics and Political Science SRC Special Paper, 1, 1-74. 